FINAL EMSP PROJECT REPORT

\title{
Rapid Mass Spectrometric DNA Diagnostics for Assessing Microbial Community Activity During Bioremediation
}

\author{
P.I.: W. Henry Benner, Lawrence Berkeley National Laboratory \\ Co-P.I.: Jennie Hunter-Cevera, Lawrence Berkeley National Laboratory \\ Co-P.I.: Joseph M. Jaklevic, Lawrence Berkeley National Laboratory \\ Collaborator: Tamas Torok, Lawrence Berkeley National Laboratory
}

\section{Executive Summary}

DNA detection and analysis has become a common way to identify organisms in many different types of samples, including soils that contain organisms potentially capable of biodegrading pollutants. Such analytical procedures, known as DNA diagnostic techniques, generally start with the extraction of DNA from organisms grown under culture conditions in the laboratory. The sequence of the DNA provides one way to identify the isolated organism. It is also possible to extract DNA directly from soil samples in an attempt to avoid the difficulty of establishing culture conditions for the large number of organisms that inhabit soils. However, DNA extracted from soil is a complicated mixture that includes DNA from all the individual organisms present in the microbial community. Several DNA amplification techniques were investigated, along with MALDI mass spectrometry, to determine how well high throughput screening procedures for tracking organisms with genes known to produce pollutant-degrading enzymes could be established.

This work focused on the identification and detection of a gene that participates in the degradation of several aromatic hydrocarbons. Biodegradation of pollutants proceeds through a number of intermediate steps that eventually culminate in an innocuous chemical. The biodegradation pathways of benzene, naphthalene, and toluene pass through catechol, an intermediate destroyed by an enzyme known as catechol dioxygenase. (Fig. 1) Thus, if DNA for the catechol dioxygenase gene is found in soil samples it is reasonable to conclude that there are organisms in the soil capable of biodegrading naphthalene,

A bacterium found to utilize naphthalene as it sole carbon source was isolated from soil. It was found to carry a gene homologous to several dioxygenase genes listed in the NCBI database. It was necessary to determine the DNA sequence of a portion of this gene because a polymerse chain reaction (PCR) product, a type of DNA diagnostic, could not be obtained from DNA extracted from this organism and with sequence found in the data base. Sequencing part of the dioxygenase gene from this organism led to the successful development of a PCR assay that could detect this gene. Conditions were then established for detecting this gene with matrix-assisted-laser desorption time-of-flight mass 
spectrometry (MALDI-TOF-MS). The most reliable mass spectrometry-based procedures are based on the detection of peptide nucleic acid probes.

Since DNA molecules extracted from soil samples could originate from any of the numerous microorganisms present in the soil, it is useful to develop procedures for studying DNA that originats only from one organism. Cloning is commonly used as a way to isolate and replicate DNA from one organism but the cloning process is sometimes inefficient due to the presence of toxic gene products and substances in soil that interfere with the biochemistry of cloning procedures.

\section{Research Objectives}

Changes in pollutant profiles observed in contaminated soils have been attributed to biological activity when in actuality abiotic processes caused pollutant removal. ${ }^{1}$ This sometimes casual implication of biodegradation is not acceptable, but understandable, because factors which cause the disappearance of pollutants are not always easy to identify. Several pollutant removal or transformation pathways are possible with most pollutants found in soil and these processes may or may not operate independently. Careful evaluation of bioremediation necessitates that all transformation and removal pathways are anticipated so that the pathways are either controlled or monitored but in practice this is generally not possible. The diminution in pollutant oxidation might track with several candidate loss mechanisms and when this occurs the real cause becomes problematic to pin point.

Many factors disguise or prevent the effectual tracking of bioremediation processes among which are three general types of problems: 1) The estimate that only about $0.1 \%$ or less of the microorganisms living in soils are culturable leads to the conclusion that the possibility for unknown organisms participating in a biodegradation pathway is high. How should these imperceptible microorganisms be tracked? 2) The diversity of the microcosm is large, yet several different genera have been shown to possess the same or very similar enzymes for degrading a pollutant. Cell counts of specific organisms, therefore may not track very well with the rate of loss or conversion of a pollutant. Should groups of organisms possessing similar biochemical reaction capabilities become the focus or is it more useful to track specific organisms? 3) Abiotic processes such as physical processes which transport pollutants away from a contamination site lead sometimes to the counterfactual conclusion that bioremediation occurred when in actuality the pollutant was diluted or dispersed heterogenously. How do we handle a large number of samples so that significantly representative sampling and monitoring strategies are implementable? Diagnostic procedures based on identifying the activity of biodegrading organisms by using DNA-based procedures are attractive in light of these problems because they can be tuned to identify groups of organisms, specific organisms, and to detect signals that measure community activity.

The ultimate desire to attribute pollutant loss to specific biochemical processes requires that the molecular degradation pathways are tracked and that all substrate or participating 
chemicals acting in the degradation pathway are present and accounted. The accounting of reactants and products, i.e. the mass balance, usually requires that different analytical tools are employed for quantitating each substance or microorganism, a daunting task in light of the complexity of most bioremediation projects.

Perhaps the best substantiation of bioremediation would be to determine a mass balance for each step in a pollutant degradation pathway and simultaneously demonstrate the involvement of an enzyme. Isolating and detecting the suspected enzyme, along with its substrate and reaction product, is further complicated because each has its own physical chemical parameters which make them difficult to isolate in a few simple steps. Enzymes are fragile and not efficiently isolated from a complex mixture of humic materials and decomposing biological debris. The indirect recognition of enzymatic activity via detection of the gene that codes for an enzyme is a preferred approach, and the application of PCR to amplify the extracted DNA, consequently augmenting sensitivity, adds further support for DNA-based methods.

The soil microbial community contains numerous different organisms ${ }^{2}$, and although diversity is desirable because different organisms participate disproportionately in degradation pathways, varying levels of diversity complicate the assessment of bioremediation $^{3}$. The issue is that other strains of a pollutant-degrading organism may or may not be able to metabolize a particular pollutant as efficiently as the more active strain. One strain might possess the capability to metabolize a pollutant but related strains are growing faster in the microcosm. For this reason, bacterial counts determined by phenotype identification is likely to be an inaccurate indicator of ongoing bioremediation. An additional problem is that bioremediation might be ongoing yet untrackable because the participating organism(s) is not easily cultured. It has been estimated that only 0.1 to 1 percent of microorganisms in soils are culturable ${ }^{4}$. Many of the nonculturable microorganisms are thought to possess the capability to degrade pollutants but their lack of growth in the lab thwarts their detection.

Finally is the issue of pollutant mixing and transport. The simple influx of clean water can leach pollutants from a region causing the local concentration to decrease but in actuality the contaminant has simply spread. Volatile solvents evaporate and the use of aeration to stimulate microorganism activity might speed up volatilization, thus disguising biological involvement. More subtle effects also are possible. Adsorption of a pollutant onto humic materials or soil particles is sometimes tenacious enough to cause their extraction, during sample processing, to become inefficient, thus leading to a purported decrease in pollutant concentration. It is possible that a pollutant adsorbs onto tiny clay particles and is spread as the particles percolate through coarse soils.

Mineralization of a pollutants could also be attributed to bioremediation when the process is actually chemical.

\section{Research Methods}

DNA diagnostic procedures are based on the determination that a specific DNA sequence is present in a sample. DNA sequence is a highly specific identification technique. DNA 
diagnostic tests can be designed to classify organisms according to genotype (using ribosomal gene markers) and at an even higher level of specificity can indicate whether an organism carries the genes to perform metabolic conversions. DNA-based diagnostic procedures have been developed in many different formats ${ }^{5}$ with target DNA ranging widely from human disease pathogens ${ }^{6}$ to extremophiles surviving in $50 \%$ toluene ${ }^{7}$. With DNA-based techniques, specific organisms are detected by sensing the presence of a DNA sequence unique to the organism ${ }^{8}$ and the successful identification of many genera of bacteria has been reported $^{9,10}$. The genes that code for pollutant degrading enzymes have also been selectively detected with the same techniques ${ }^{11,12,13}$. Regardless of the source of the DNA, the ability to identify the presence of a target sequence forms the basis of DNA diagnostic procedures.

As related to the focus of this research project, the determination that a target sequence is present in a sample is used to indicate that the dioxygenase gene is carried by the organisms under investigation. In addition to the specificity of the diagnostic test, another compelling advantage of DNA-based diagnostic tests is sensitivity. Under appropriate conditions, DNA molecules can be enzymatically replicated in order to enhance the concentration of the target molecules without the requirement of sample-concentrating analytical procedures that are typical of most other analytical schemes. A relatively small number of targeted DNA molecules can be replicated (amplified) to generate picomoles of copied DNA with the polymerase chain reaction (PCR).

The way the tests are scored depends on the design of the test. In a PCR-based assay, primer molecules of DNA oligonucleotides define the targeted DNA. . Judicious choice of PCR primers leads to the amplification of a targeted sequence and the number of bases in the amplified DNA, normally determined with gel electrophoresis, serves as diagnostic step. If the PCR reaction produced a product with an expected size, then the diagnostic is scored as positive. The use of random priming sites ${ }^{14}$ is also possible but the length of the PCR product(s) may not be known until control experiments are run and the reaction products are separated on a gel. Both priming strategies have been used to design diagnostic procedures and the size of the PCR products establishes a database that is useful for identifying microorganisms.

In a related diagnostic procedure the PCR product is digested with a restriction enzyme(s) to produce shorter fragments that have a sequence dependent polymorphism. This supplies the basis of DNA fingerprinting ${ }^{15,16,17,18}$, an approach that provides more information than the size of the PCR product alone. If the enzyme cuts at locations where the sequence shows variation between organisms, then the gel banding pattern will reveal sequence specific fragment lengths unique to a group of organisms. The size of the restriction fragments can also be used to generate a database for identifying organisms.

A third type of diagnostic approach utilizes target DNA, such as that isolated from cultures or PCR reactions, applies the oligonucleotide ligation assay as a final step in the procedure. ${ }^{19,20}$ Instead of separating the PCR reaction products on a gel, the amplified DNA is denatured and exposed to two oligonucleotides designed to be complementary 
and abutting after they anneal to a short sequence in the target DNA. In this approach, the sequence of the target must be known so that complementary oligonucleotides can be chosen and synthesized. If the two oligonucleotides anneal to the target and lie abuttingly, than ligase will join them together. If the oligonucleotides are not abutting, then ligase can not join them together. The basis of this type of diagnostic procedure relies on confirming the formation of the ligated oligonucleotides. Another form of this diagnostic procedure uses a sequential reaction called the ligation chain reaction $(\mathrm{LCR})^{21,22}$, a technique introduced to enhance sensitivity.

Matrix-assisted-laser-desorption-ionization (MALDI) time-of-flight mass spectrometry was evaluated as a way to score oligonucleotide ligation assays (OLA). The application of mass spectrometry to the analysis of amplified DNA is an attractive alternative to gelbased procedures for three reasons. 1) Gel separations are time consuming and the pouring, loading, staining, visualization, and molecular size assessment is labor intensive. Improved throughput can be expected for mass spectrometry detection because of the speed with which mass spectrometry operates. A mass spectrum is collected in seconds to minutes thus significantly shortening running time. Time-of-flight mass spectra are generated in digital form allowing them to be saved in computer memory, and if needed, processed later. The mass spectrometry approach avoids staining and visualization procedures normally used after DNA has been separated with a gel and avoids expensive reagents used in OLA reactions. Similar improvements are expected in the Human Genome Center at LBNL where we are attempting to solve several analytical bottlenecks by introducing advanced mass spectrometry methods into genome mapping procedures. 2) The operation of gels is not easily automated. A few of the steps in the process have been recently automated and implemented into the Human Genome Center at LBNL but many steps are still laborious. Gel casting and staining have not been automated. Gel loading and gel visualization are now performed nearly automatically, but still require constant operator attention and an attendant is needed to move gels from one instrument to another. In contrast, PCR samples can be injected directly into the ion source of an electrospray mass spectrometer with minimal processing. Matrix-assisted-laserdesorption time-of-flight mass spectrometry (MALDI) samples are prepared by evaporating $1 \mu \mathrm{L}$ of sample along with $1 \mu \mathrm{L}$ of matrix solution on the end of a MALDI probe. Commercially available MALDI source plates have recently been introduced which provide a way to introduce collectively many samples into the vacuum chamber. The spotting and evaporation of samples onto a MALDI sample holder appears to be relatively easy to automate. 3) Theoretically, mass spectrometry is capable of high sensitivity.

\section{Research Results/Findings}

To determine the optimal regions of the dioxygenase gene on which to focus the diagnostic tests, a survey of the database of the National Center for Biotechnology Information (NCBI) was conducted. From the survey, it was determined that there were several areas of both protein and nucleotide sequence similarity among several bacteria which are able to metabolize naphthalene. The best areas of sequence similarity were 
found on the nahC (2-dihydroxynaphthalene dioxygenase) and nahH (catechol 2,3dioxygenase) genes.

To obtain the exact sequence of a region of interest of the naphthalene-degrading genes, a sample of DNA from Pseudomonas stutzeri P-16 was obtained. P-16 was isolated by W. T. Stringfellow (LBNL) from soil contaminated by creosote. The bacterial strain was tested and found to be able to grow using naphthalene as its sole source of carbon.

Using rough sequence data from the NCBI database, DNA primers were synthesized and a 161-base pair region of the nahH gene on P-16 was amplified by means of PCR. The product was cloned into a vector and transformed into E. coli cells with the aid of a Stratagene cloning kit. The cells were allowed to grow for several days, after which DNA was recovered from the cells by means of a Qiagen mini-prep kit. The DNA was purified and then sequenced, shown in Fig. 2, on an ABI sequencer.

From the sequence obtained for the 161-base pair region of the nahH gene of P-16, primers were designed and synthesized for an LCR reaction. Four primers approximately 20 bases in length were constructed such that two would lie abuttingly on the sense strand of the P-16 template DNA and that the other two would lie abuttingly on the antisense strand. Once ligated together, the sense 40-base product would be complementary to the anti-sense product. The actual points of ligation, however, were offset by one base to reduce the probability of primers not annealed to the appropriate template to be ligated together. Primers were designed to have relatively high melting temperature to increase reaction specificity, but were also designed to produce a low-melting point product to facilitate efficient denaturation at the high temperature point of each cycle. To one primer was attached a biotin label for use in both detection assays.

Primers were added to a solution of template DNA, T4 ligase, an appropriate buffer, and salmon sperm DNA as an deterent to non-specific annealling. The reaction mixture was cycled between high (denaturation) and low (annealing and ligation) temperatures 32 time in a Perkin-Elmer 9700 thermocycler. The reagent concentrations and thermocycling parameters were optimized to minimize the amount of ligation observed in negative control reactions (reactions in which no template DNA was added) and to maximize the amount of ligation observed in positive control reactions.

Initially, LCR reactions were scored as positive or negative by means of an Oligonucleotide Ligation Assay (OLA), supplied with the LCR kit obtained from BioRad. In the OLA, the biotin label of the LCR product binds to the bottom of a streptavidin-coated microtiter plate. Through a series of wash and incubation steps, the LCR product was treated in such a way that if the ligation reaction was successful to a large degree, a reaction producing a solution color change would occur. If ligation was not successful, no color change would be observed. From the OLA, it was learned that initial primer concentrations had to be kept extremely low ( $<600 \mathrm{fmol} / 50 \mathrm{ul}$ reaction) in order to prevent false positive reactions from occuring. 
The other assay used to score LCR reactions was mass spectrometry based. The biotin label of the LCR products was bound to streptavidin-coated magnetic beads. The LCR products were washed and the denatured with ammonium hydroxide. The nonbiotinylated strand of LCR product was recovered, purified, and then analyzed by MALDI-TOF MS. If the ligation was successful, a ligation product of 40 bases, corresponding to a mass of approximately $13000 \mathrm{Da}$, would appear on the mass spectrum. If the ligation was not successful, an unligated primer of 20 bases, or around 6 $500 \mathrm{Da}$, would appear on the mass spectrum.

Because reagent concentrations were so dilute to prevent false positive signals, the products were too dilute to be seen reliably in mass spectra. The mass spectrometry technique was sensitive enough to register 20-base negative reaction signals at concentrations of $500 \mathrm{fmol} / \mathrm{ul}$, but it was not sensitive enough to register 40-base positive reaction signals at similar concentrations. In addition, sample recovery in the affinity capture, wash, and denaturation steps was estimated to be less than $70 \%$, making observation of LCR products by MALDI-TOF MS less reliable.

Using the sequence obtained from the nahH gene of $\mathrm{P}-16$, another set of PCR primers were designed and synthesized. These primers were designed to PCR-amplify an 84-base pair region of the gene to be subsequently analyzed by a peptide nucleic acid (PNA) probe. The PCR primers were designed to have very high melting temperatures to improve reaction specificity. One primer had a biotin label attached for use in the probe assay. A PNA probe was also designed and synthesized. The probe was designed to be complementary to a 14-base region of the biotinylated strand of the PCR product.

PCR reactions were conducted using the new primers, P-16 as template DNA, glycerol as a cosolvent, Ampli-Taq Gold DNA polymerase from Perkin-Elmer, and an appropriate buffer. Reagent mixing was carried out in a carefully cleaned environment to prevent contamination of the reagents or reaction mixtures by stray pieces of DNA. Glycerol was used in the reaction to improve annealing specificity of the primers to the template. Taq Gold polymerase was used as a safeguard against false amplification during the initial temperature ramp of the thermocycler. Reactions were carried out in a Perkin-Elmer 9700 thermocycler. The PCR tubes were cycled through 40 repetitions of high (denaturation) and low (annealing and extention) temperatures. The presence or absence of the expected 84-base pair product was confirmed by agarose gel electrophoresis.

PCR product samples were prepared for mass spectrometry analysis by purifying them to remove salts and unincorporated primers from the solution. The products were then attached to streptavidin-coated magnetic beads by means of the biotin label on one PCR primer. The beads were washed several times, and then the PCR product was denatured with sodium hydroxide. The non-biotinylated strand was washed away after which the PNA probe was introduced and allowed to anneal to the biotinylated strand of DNA, still attached to the magnetic beads. The beads were again washed several times to eliminate as much sodium from the solution as possible and then were analyzed by MALDI-TOF MS. If the PCR reaction was successful, PNA probes would bind to the region of interest of the PCR product and would subsequently appear a a peak in a mass spectrum. If the 
PCR reaction was negative, however, no PCR products would be produced and the probe would have nothing to which to bind. In that case, no signal would be seen in a mass spectrum.

The mass spectrometer was able to analyze PNA probes while they were still hybridized to the complementary strand of DNA. A sample of beads was combined with a matrix solution of SA matrix and tri-fluoroacetic acid (TFA) and allowed to crystallize onto the MALDI sample holder. The sample preparation steps are shown in Fig. 4. The sample was ionized by a $337 \mathrm{~nm}$ nitrogen laser. The PNA probes detached from their complementary DNA either during the crystallization or ionization process, which allowed it to appear at its own mass value in the spectrum. (See Fig. 5.) The spectrometer was sensitive enough to see PNA probes in solutions as dilute as 500 fmol/ul. There was a strong correlation between concentration and peak height for PNA probes ionized and observed under identical conditions. There was also a strong correlation between peak height and amount of PCR product available for hybridization, as determined by band intensity on an agarose electrophoresis gel. The mass spectrometric analysis was more sensitive than gel analysis by a factor of ten. In electrophoresis gels, PCR product could only be observed when at least 30000 copies of template P-16 were in the PCR reaction tube whereas PNA probes were observed in spectra from PCR reactions that only contained 3000 copies of template DNA.

To further evaluate this method of DNA diagnostics on bacterial samples other than P-16, samples of two strains of bacteria known to degrade polycyclic aromatic hydrocarbons were obtained from Julia Foght, et. al., University of Alberta, Canada. These bacteria were grown in our laboratory using naphthalene or phenanthrene as a sole carbon source. DNA was obtained from one of these bacterial strains by means of a mini-prep. PCR was conducted using this bacterial DNA as template but using the same primers designed for P-16. Gel analysis confirmed the presence of an 84-base pair PCR product, indicating that the bacterium did in fact contain the nahH gene for which we were probing.

\section{Personnel Supported}

H. Benner, P.I. , project oversight, mass spectrometry technique development supervision J. Hunter-Cevera, supervision of microbiology activities

C. Hack, biology technician

W. Searles, instrument maintenance, hardware development for MALDI and CDMS

S. Goldman, molecular biologists for procedure development

S. Ferriero, machinist for instrument development

E. Cornell, programming for data analysis

E. Jackson, coop student form UCD

R. Thomas, coop student from UDB, MALDI sample analysis

\section{Transition}

Advised Ion Diagnostics, Inc. on the feasibility of DNA technology in their desire to enter the diagnostics business 


\section{Patents, none}

\section{Future Work}

The results demonstrated the analytical approach determines the presence of a specific gene in cellular extracts. The next step will be to evaluate this technique using DNA extracted from soil.

\section{Figures}




\section{Importance of dioxygenase gene}

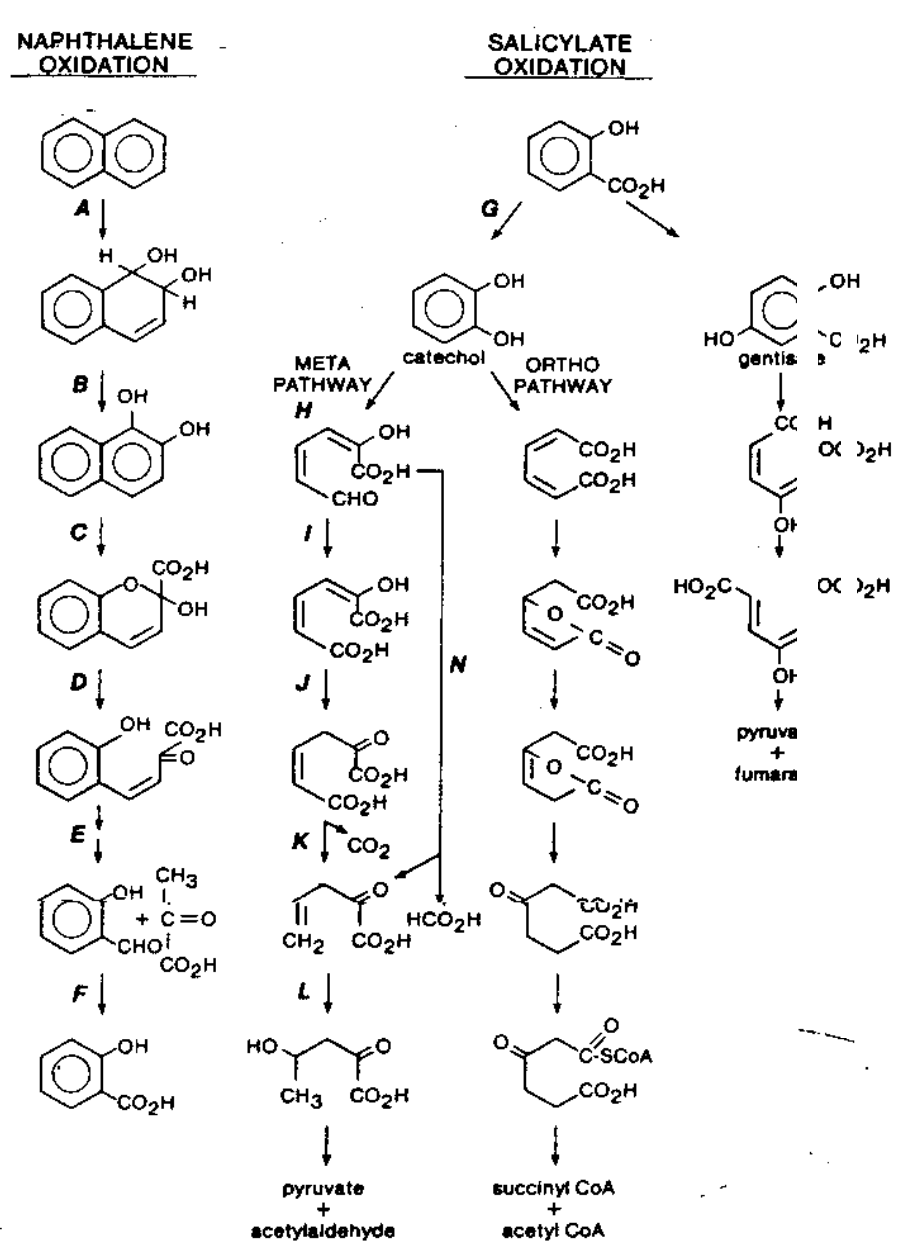

Figure 1. Benzene, Naphthalene and Toluene degradation pathway involving the dioxygenase gene for catechol 


\section{Sequence Obtained for the Naphthalene dioxygenase Gene}

P-16: Pseudomonas stutzeri $\mathrm{P}-16$, sequenced in-house

Underlined region

Ppu: Pseudomonas putida database sequence

denotes PCR amplicon

$\begin{array}{cc}\text { P-16 } & \text { PNA probe } \\ \text { Ppu } & \text { TtgCCGTGAGTGAGCCGTGCGGTTGGGCGATGTCGATCGAG } \\ \text { P-16 } & \text { GTGTCGGTCATGGAGATCAGGTCGGCGCCCGGAGCACGTCTTCC } \\ \text { Ppu } & \text { GTGTCGGTCATGGAGATCAGGTCGGCGGCGCGAAGCACGTCTTCC } \\ \text { P-16 CAACTCTCTAGGAAGAATGAAGCGCGATGGAATCGGCCCTTCTCC } \\ \text { Ppu CAGGTTTCGAGGTGGAAGGACACATGATGGAGGCGGCCTTTTCC } \\ \text { P-16 GAATGATGAATGAAGGCCACGTCGTGCGCTTTGGTG--------- } \\ \text { Ppu GGATGGTGAATGAAGGCCACGTCGTGGGCCTTGGTCGACAGGCTG }\end{array}$

Figure 2. DNA sequence (partial) of the dioxygenase gene 


\section{Identification of Bacterial DNA}

\section{Case 1: Bacterial DNA contains gene of interest}

1. PCR amplify region of bacterial DNA

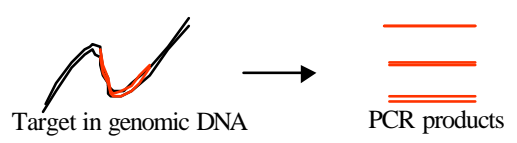

2. Perform PNA affinity capture assay (PNA binds to PCR amplicon)

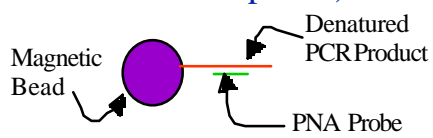

3. Analyze by MALDI-TOF MS

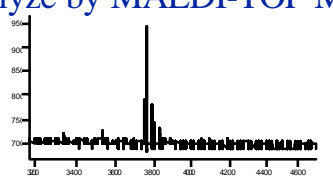

4. Spectrum shows PNA peak; test scored positive
Case 2: Bacterial DNA does not contain gene of interest

1. Attempt PCR of bacterial DNA (no amplicon produced)

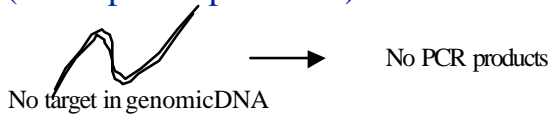

2. Perform PNA affinity capture assay (PNA does not bind and is washed away)

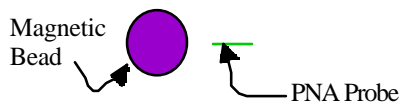

3. Analyze by MALDI-TOF MS

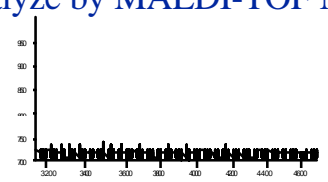

4. Spectrum shows no PNA peak; test scored negative

Figure 3. Methodology for using MALDI-TOF-MS for scoring PCR or LCR reaction products 


\section{MALDI Sample Preparation for Bead- DNA-PNA Complex}

\begin{tabular}{|c|c|c|c|c|}
\hline $\begin{array}{c}\text { Spect. } \\
\#\end{array}$ & $\begin{array}{c}\text { Matrix } \\
\text { Solution }\end{array}$ & $\begin{array}{c}\text { PH of } \\
\text { Matrix } \\
\text { Solution }\end{array}$ & $\begin{array}{c}\text { Sample } \\
\text { Prep. } \\
\text { Temperture }\end{array}$ & $\begin{array}{c}\text { PNA }^{+} \\
\text {Intensity }\end{array}$ \\
\hline A & $\begin{array}{c}\text { SA in 2:1 } \\
\mathrm{H}_{2} \mathrm{O} / \mathrm{ACN}\end{array}$ & $3 \sim 4$ & $\begin{array}{c}\text { Room } \\
\text { Temperture }\end{array}$ & 0 \\
\hline $\mathrm{B}$ & $\begin{array}{c}\text { SA in 2:1 } \\
\mathrm{H}_{2} \mathrm{O} / \mathrm{ACN}\end{array}$ & $3 \sim 4$ & $\begin{array}{c}\text { incubate @ } \\
76^{\circ} \mathrm{C} \text { for 2 } \\
\text { min. }\end{array}$ & 50 \\
\hline $\mathrm{C}$ & $\begin{array}{c}\text { SA in 2:1 } \\
0.1 \% \\
\text { TFA/ACN }\end{array}$ & $>1$ & $\begin{array}{c}\text { Room } \\
\text { Temperture }\end{array}$ & 200 \\
\hline
\end{tabular}

Figure 4. Sample preparation for MALDI-TOF-MS of PNA probes. 


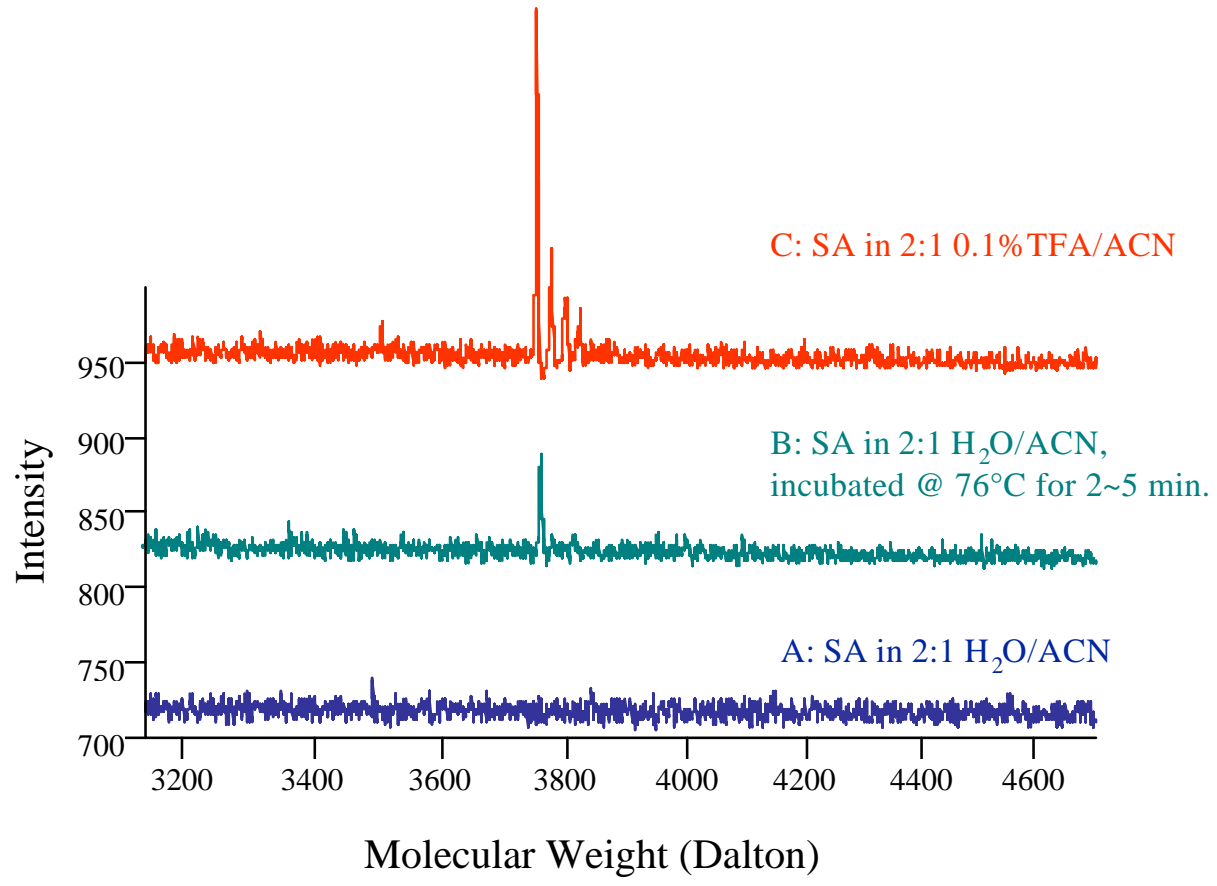

Figure 5. MALDI-TOF-MS spectra of PNA probes. See Fig. 4 for explanation of labels. 


\section{Literature Cited}

\footnotetext{
${ }^{1}$ Shannon, M.J.R. and Unterman R., "Evaluating Bioremediation: Distinguishing Fact from Fiction," Annu. Rev. Microbiology, 47, 715-738, (1993).

${ }^{2}$ Stim, K.P., A phylogenetic analysis of micro-organisms isolated from subsurface environments, Molecular Ecology, 3, 1-10, 1994.

${ }^{3}$ Reber H.H., "Simultaneous Estimates of the Diversity and the Degradative Capability of Heavy-metalaffected Soil Bacterial Communities," Biology \& Fertility of Soils, 13, 181-186 (1992).

${ }^{4}$ Allas R.M. and Bartha R., "Microbial Ecology," 2nd Ed., The Benjamin Cummings Pub. Co., Reading, MA (1987).

${ }^{5}$ Species Diagnotic Protocols, PCR and Other Nucleic Acid Methods, J.P. Clapp (Ed.), Methods in Molecular Biology, No. 50, Humana Press, Totowy, NJ (1996).

${ }^{6}$ Greisen,K., M. Loeffelholz, A. Purohit and D Leong, PCR primers and probes for the 16S rRNA gene of most species of pathogenis bacteria, including bacteria found in cerebrospinal fluid, J. Clin. Microbiol. 32, 335-351, 1994.

7 Atlas, R.M., Bioremediation, Chem. and Enging. News, April 3, 1995 , p32.

${ }^{8}$ Brockman F.J., Hinchee R.E., G.S. Douglas and Ong S.K (Ed), "Overview of Biomolecular Methods for Monitoring Bioremediation Performances in Monitoring and Verification of Bioremediation," Battelle Press, Columbus, OH (1995).

${ }^{9}$ Jiang H.Y. and Sato K., "DNA Base Composition of Bacterial Groups Differentiated by a Diagnostic Scheme Used for Soil Isolates," Soil Sci. Plant Nutr., 40, 675-681 (1994).

${ }^{10}$ Greisen K., Loeffelnolz, Purohit A. and Leong D., "PCR Primers and Probes for the IGS RNA Gene of Most Species for Pathogenic Bacteria, including Bacterial Found in Cerebrospinal Fluid,” J. Clin.

Microbiol., 32, 335-351, (1994).

${ }^{11}$ Stehan R.J. and Atlas R.M., Polymerase Chain Reaction: Applications to Environmental Microbiology, “ Annu. Rev. Microbiol, 45, 137-161 (1991).

${ }^{12}$ Ka J.O, Holben W.E. and Tiedje J.M., "Use of Gene Probes to Aid in Recovery and Identification of Functionally Dominant 2,4 Dichlorophenoxy Acetic Acid-degrading Populations in Soil, Appl. Environ. Microbiol. 60, 1116-1120 (1994).

${ }^{13}$ Erb R.W. and Wagner-Döbler I, "Detection of Polychlorinated Biphenyl Degradation Genes in Polluted Sediments by Direct DNA Extraction and Polymerase Chain Reaction," Appl. Environ. Microbiol., 59, 4065-4073 (1993).

${ }^{14}$ Bazzicalupo, M. and Fani R., "The Use of RAPD for Generating Specific DNA Probes for Microorganisms, in Species Diagnostics Protocols, Methods in Molecular Biology, No. 50, Humana Press, Totowa, NJ, (1996).

${ }^{15}$ Grattard F., Etienne J., Pozzetto B., Tandy F., Gauderi O.G. and Fleurette J., "Characterization of Unrelated Strains of Staphylococcus Schleiferi Using Ribosomal DNA Fingerprinting, DNA Restriction Patterns, and Plasmid Profiles," J. Clin. Microbiol., 31, 812-818 (1993).

${ }^{16}$ Seifert H., Schulze A., Baginski R. and Pulverer G., "Plasmid DNA Fingerprinting of Acinetobacter Species and Other than Acinetobacter Baumannii," J. Clin. Microbiol., 32, 82-86 (1994).

${ }^{17}$ Forbes K.J., Bruce K.D., Jordens J.Z., Ball A. and Pennington T.H., J. Gen. Microbiol., 137, 2051-2058 (1991).

${ }^{18}$ Bassam B.J., Caetano-Anollés G. and Gresshoff M., "DNA Complication Fingerprinting of Bacteria," Appl. Microbiol. Biotechnol. 38, 70-76 (1992).

${ }^{19}$ Nickerson D.A., Kaiser R., Lappin S., Stewart J., Hood L. and Landegren U., Proc. Natl Acad. Sci., 87, 8923-8927 (1990).

${ }^{20}$ Landegren U., Kaiser R., Sanders J. and Hood L., “A Ligase-mediated Gene Detection Method,” Science, 241, 1077-1080 (1988).

${ }^{21}$ Widemann M., Wilson W.J., Czajka J., Luo J., Barany F. and Batt C.A., "Ligase Chain Reaction (LCR) Overview and Applications in PCR Methods and Applications, "Cold Spring Harbor Laboratory (1994).

${ }^{22}$ Wu D.Y. and Wallace B., "The Ligation Amplification Reaction (LAR). Amplification of Specific DNA Sequences Using Sequential Rounds of Template-dependent Ligation,” Genomics, 4, 560-569 (1989).
} 